\title{
Fatores associados à insegurança alimentar e nutricional em comunidade carente
}

\section{Factors associated with food and nutrition insecurity in a needy community \\ Factores asociados con la inseguridad alimentaria y nutricional de una comunidad desfavorecida}

\author{
Andressa Pestana Brito (iD \\ Universidade Ceuma - São Luís (MA) - Brasil \\ Virgínia Nunes Lima iD \\ Faculdade Pitágoras - São Luís (MA) - Brasil \\ Eliziane Gomes da Costa Moura da Silva (iD \\ Universidade Ceuma - São Luís (MA) - Brasil

\section{Adriana Sousa Rêgo iD} \\ Universidade Ceuma - São Luís (MA) - Brasil \\ Luciana Pereira Pinto Dias (iD \\ Universidade Ceuma - São Luís (MA) - Brasil \\ Josélia Dias Silva (iD \\ Universidade Ceuma - São Luís (MA) - Brasil
}

\section{Wyllyane Rayana Chaves Carvalho (iD}

Instituto Florence - São Luís (MA) - Brasil

Janaina Maiana Abreu Barbosa (iD

Universidade Ceuma - São Luís (MA) - Brasil

\section{RESUMO}

Objetivo: Verificar os fatores associados à insegurança alimentar e nutricional (IAN) de uma comunidade carente. Métodos: Estudo transversal e analítico, desenvolvido nos meses de agosto e setembro de 2018, com 150 indivíduos, em uma comunidade carente de São Luís, Maranhão, Brasil. Participaram da pesquisa maiores de 18 anos, de ambos os sexos e residentes em domicílios na comunidade escolhida. Não foi incluído mais de um indivíduo por domicílio. Aplicou-se um questionário para obtenção de dados sociodemográficos (sexo, idade, escolaridade, renda, cor da pele, número de moradores da residência, estado civil e ocupação). A situação de insegurança alimentar e nutricional foi avaliada com auxílio da Escala Brasileira de Insegurança Alimentar, que mede a percepção e a experiência familiar da segurança alimentar, insegurança e fome em nível domiciliar, classificando o domicílio em segurança ou insegurança alimentar (leve, moderada ou grave). Realizou-se análise multivariada pelo modelo de regressão de Poisson. Resultados: A prevalência de IAN foi de $94 \%(n=141)$ nos avaliados. No que se refere à renda, $70,7 \%(n=106)$ tinham renda mensal menor que um salário mínimo. Quanto à escolaridade, $50,7 \%(n=76)$ relataram tempo inferior a oito anos de estudo. Observou-se associação em ter renda familiar menor que um salário mínimo (IRR=1,07; IC=1,07-1,31) com a IAN. Conclusão: A prevalência de insegurança alimentar foi elevada entre os indivíduos investigados e mostrou-se associada à menor renda.

Descritores: Segurança Alimentar e Nutricional; Fatores de Risco; Vulnerabilidade Social.

\section{ABSTRACT}

Objective: To verify the factors associated with Food and Nutrition Insecurity (FNI) in a needy community. Methods: This analytical cross-sectional study was conducted from August to September 2018 with 150 people from a needy community in São Luís, Maranhão, Brazil. Participants were 18 years old, of both sexes, and lived in households in the chosen community. No more than one person per household was included. A questionnaire was used to obtain sociodemographic data (sex, age, education, income,

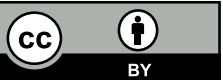


skin color, number of people living in the household, marital status and occupation). Food and nutrition insecurity status was assessed by the Brazilian Food Insecurity Scale, which measures family's perception and experience of food security, insecurity and hunger in the household and classifies the household as either food security or food insecurity (mild, moderate or severe). Multivariate analysis was performed using the Poisson regression model. Results: The prevalence rate of $F N I$ was $94 \%$ ( $n=141$ ) in the people analyzed. Regarding income, $70.7 \%(n=106)$ of the participants had a monthly income of less than one minimum wage. As for education, $50.7 \%(n=76)$ of the participants reported having less than eight years of study. There was an association between household income of less than one minimum wage (IRR=1.07; CI=1.07-1.31) and FNI. Conclusion: The prevalence of food insecurity was high among the people analyzed and it was associated with lower income.

Descriptors: Food and Nutrition Security; Risk Factors; Social Vulnerability.

\section{RESUMEN}

Objetivo: Verificar los factores asociados con la inseguridad alimentaria y nutricional (IAN) de una comunidad desfavorecida. Métodos: Estudio transversal y analítico desarrollado en los meses entre agosto y septiembre de 2018 con 150 individuos de una comunidad desfavorecida de São Luís, Maranhão, Brasil. Mayores de 18 años, de ambos los sexos y residentes de domicilios de la comunidad elegida participaron de la investigación. No se ha incluido más de uno individuo de cada domicilio. Se aplicó una encuesta para obtener los datos sociodemográficos (el sexo, la edad, la escolaridad, la renta, el color de la piel, el número de personas de la vivienda, el estado civil y la ocupación). La situación de inseguridad alimentaria y nutricional ha sido evaluada por la Escala Brasileña de Inseguridad Alimentaria que mide la percepción y la experiencia de la familia sobre la seguridad alimentaria, la inseguridad y el hambre a nivel domiciliario clasificándole de seguridad o inseguridad alimentaria (leve, moderada o grave). Se realizó un análisis multivariado por el modelo de regresión de Poisson. Resultados: La prevalencia de IAN en los evaluados ha sido del $94 \%$ (n=141). Sobre la renta, el 70,7\% $(n=106)$ tenía la renta mensual de menos de un sueldo mínimo. Respecto la escolaridad, el 50,7\% $(n=76)$ ha relatado haber tenido menos de ocho años de estudio. Se observó la asociación entre tener la renta familiar de menos de un sueldo mínimo (IRR=1,07; IC=1,07-1,31) y la IAN. Conclusión: La prevalencia de inseguridad alimentaria ha sido elevada entre los individuos investigados y se ha asociado con la renta baja.

Descriptores: Seguridad Alimentaria y Nutricional; Factores de Riesgo; Vulnerabilidad Social.

\section{INTRODUÇÃO}

A insegurança alimentar e nutricional (IAN) ocorre quando uma família não consegue realizar, de forma regular e permanente, a aquisição de alimentos de qualidade e em quantidade suficiente ou quando, para tê-lo, compromete o acesso a outras necessidades essenciais ${ }^{(1)}$. A IAN incide na sensação de preocupação e/ou angústia diante da incerteza da disponibilidade do alimento diariamente, na convivência com a fome e no consumo de uma dieta de baixa qualidade nutricional, monótona e insuficiente para suprir as necessidades de uma pessoa ${ }^{(2)}$.

A estimativa do Brasil, mostrada na Pesquisa Nacional por Amostra de Domicílio (PNAD) de 2013, indicou o Nordeste como a região de maior taxa de domicílios em situação de IAN, 45 milhões de moradores, o que equivale a $38,1 \%$ dos domicílios nordestinos avaliados ${ }^{(3)}$, e o Maranhão foi o estado que registrou a maior prevalência de IAN (60,9\%). Esse cenário mostra a dificuldade de acesso regular e permanente a alimentos com qualidade e em quantidades suficientes nesse estado ${ }^{(4)}$.

De acordo com o Instituto Brasileiro de Geografia e Estatística (IBGE), o Índice de Desenvolvimento Humano (IDH) de São Luís, Maranhão, demostrou significativa evolução entre os anos de 2000 e 2010 (0,658 e 0,768), mas ainda apresenta desigualdade de renda, problemas estruturais e pessoas que vivem na linha da pobreza( ${ }^{(5)}$. Essa vulnerabilidade apresentada pela população maranhense pode ser explicada pela situação de pobreza e desigualdade social que ainda atinge o estado, além de outros fatores socioeconômicos e demográficos que, segundo relatos, estão associados à IAN de uma população, como renda familiar, número de moradores residentes no domicílio, sexo, cor da pele, escolaridade, local de residência e presença/ausência de menores de 18 anos de idade no domicílio(6-9).

A IAN está associada à pobreza e à extrema pobreza em que os indivíduos vivem, a qual pode ser medida por cinco métodos de análise da situação de segurança alimentar e nutricional (SAN), comumente empregados em inquéritos nacionais. Entre esses métodos, destacam-se as escalas psicométricas do acesso familiar aos alimentos, como a Escala Brasileira de Insegurança Alimentar (EBIA) ${ }^{(10)}$.

A SAN, segundo a Política Nacional de Segurança Alimentar e Nutricional (PNSAN), por meio da Lei n. ${ }^{0} 11.346$, de 15 de setembro de 2006, conhecida como Lei Orgânica de Segurança Alimentar e Nutricional (LOSAN), pode ser definida como o direito de todos ao acesso regular e permanente a alimentos de qualidade e em quantidade suficiente sem prejuízo ao acesso a outras necessidades básicas, como educação e lazer, respeitando a diversidade cultural, social, econômica e ambientalmente sustentáveis ${ }^{(11)}$. 
A alimentação constitui um dos direitos humanos básicos, representando um requisito para a corroboração de todos os outros direitos inerentes ao próprio conceito e exercício de cidadania. Abrange aspectos que não se limitam apenas ao acesso aos alimentos em si, mas ao próprio contexto em que pessoas e comunidades estão inseridas, cabendo ao Estado a fomentação e garantia de sua observação, de modo a configurar uma prerrogativa essencial de todo cidadão de não sentir receio de viver sob a ameaça de fome ${ }^{(12)}$.

Portanto, com o objetivo de garantir uma alimentação saudável e adequada a todos, o Brasil faz uso de instrumentos que estão inseridos na Política Nacional de Promoção da Saúde através de atividades que envolvem a alimentação saudável e promoção da saúde e a SAN, apoiando ações e metas para a diminuição da pobreza, maior abrangência social e implementação do direito humano à alimentação adequada (DHAA) ${ }^{(13)}$.

Conhecer a prevalência da IAN é de grande importância para a avaliação das condições de vida, pois o quadro de IAN é preocupante e estudos nessa área poderão subsidiar a criação de políticas públicas para a superação do problema. Diante do exposto, este estudo objetivou verificar os fatores associados à insegurança alimentar e nutricional (IAN) de uma comunidade carente.

\section{MÉTODOS}

Estudo transversal e analítico, realizado durante os meses de agosto e setembro de 2018, em uma comunidade carente de São Luís, Maranhão, Brasil. Trata-se de uma comunidade da zona urbana localizada na periferia da capital. A amostra do tipo não probabilística foi composta por 150 indivíduos que estavam nos seus domicílios no momento da coleta de dados e que aceitaram participar da pesquisa.

Participaram da pesquisa pessoas maiores de 18 anos, de ambos os sexos, residentes em domicílios na comunidade escolhida para o estudo e que aceitaram assinar o Termo de Consentimento Livre e Esclarecido (TCLE). Não foi incluído mais de um indivíduo por domicílio.

Realizou-se a aplicação da coleta de dados por meio de entrevista com os residentes nos domicílios em estudo. Utilizou-se um questionário adaptado ${ }^{(14)}$ contendo questões relacionadas às variáveis sociodemográficas (sexo, idade, escolaridade, renda, cor da pele, número de moradores da residência, estado civil e ocupação).

O segundo instrumento aplicado foi a Escala Brasileira de Insegurança Alimentar (EBIA), com 15 questões fechadas, aplicada pelo IBGE na PNAD realizada nos anos de 2004, 2009 e 2013 para mensurar a prevalência de domicílios com IAN no país. Ressalta-se que a PNAD é a única pesquisa representativa do Brasil com dados disponíveis sobre IAN ${ }^{(15)}$. A EBIA é considerada um instrumento de baixo custo e de fácil aplicação, e mede a percepção e a experiência familiar da segurança alimentar, insegurança alimentar e fome em nível domiciliar, tornando possível a estimativa de sua prevalência na população( ${ }^{(4)}$, classificando o domicílio em análise em segurança ou insegurança alimentar, em três níveis: leve, moderado e grave ${ }^{(16)}$.

A metodologia da EBIA recomenda a construção de um escore, atribuindo um ponto para cada resposta positiva. Se o domicílio obtém pontuação zero, é classificado em SAN. Existem duas pontuações diferentes referentes à classificação de IAN para domicílios com e sem residentes menores de 18 anos. Assim, quando o domicílio tem apenas moradores maiores de 18 anos, a classificação é: de 1 a 3 pontos - insegurança alimentar leve (IAL); de 4 a 6 pontos - insegurança alimentar moderada (IAM); de 7 a 8 pontos - insegurança alimentar grave (IAG). Nos domicílios com menores de 18 anos de idade, a classificação dos níveis de IAL, IAM ou IAG corresponde a 1 a 5 pontos, 6 a 10 pontos e 11 a 15 pontos, respectivamente ${ }^{(4)}$. Os dados coletados foram tabulados e organizados no programa Microsoft Office Exce/®, versão 2010, e posteriormente transferidos para o software STATA $\Theta$, versão 12.0, no qual se realizaram as análises, sendo apresentadas as variáveis categóricas por meio de frequências absolutas e relativas.

Posteriormente, realizou-se análise multivariada pelo modelo de regressão de Poisson, sendo incluídas no modelo múltiplo todas as variáveis independentes (sociodemográficas) associadas ao evento de interesse (IAN), com significância estatística de até $20 \%$. Para aceitação das associações investigadas no modelo final, adotou-se o valor de $p<0,05$.

O presente estudo faz parte de um projeto guarda-chuva aprovado pelo Comitê de Ética em Pesquisa da Universidade Ceuma com o Parecer consubstanciado n. ${ }^{\circ} 2.627 .604$.

\section{RESULTADOS}

Dos 150 indivíduos entrevistados, 68,7\% ( $n=103)$ eram do sexo feminino, 54,7\% ( $n=82)$ viviam com companheiro e $44 \%(n=66)$ referiram estar desempregados. As residências com moradores de 18 a 29 anos representaram $50 \%$ 
$(n=75)$ da amostra. No que se refere à renda, 70,7\% $(n=106)$ tinham renda mensal menor que um salário mínimo. Mais da metade dos indivíduos $(54,7 \% ; n=82)$ moravam em domicílios compostos por um ou três membros. Quanto à escolaridade, $50,7 \%(n=76)$ relataram tempo inferior a oito anos de estudo. De acordo com a autodeterminação da raça, $46,7 \%(n=70)$ se declararam pardos (Tabela I).

Tabela I - Distribuição dos participantes segundo as condições sociodemográficas. São Luís, Maranhão, 2019.

\begin{tabular}{|c|c|c|}
\hline Variável & $\mathbf{n}$ & $\%$ \\
\hline \multicolumn{3}{|l|}{ Sexo } \\
\hline Masculino & 47 & 31,3 \\
\hline Feminino & 103 & 68,7 \\
\hline \multicolumn{3}{|l|}{ Estado Civil } \\
\hline Com companheiro(a) & 82 & 54,7 \\
\hline Sem companheiro (a) & 68 & 45,3 \\
\hline \multicolumn{3}{|l|}{ Idade (anos) } \\
\hline $18-29$ & 75 & 50,0 \\
\hline $30-59$ & 55 & 36,7 \\
\hline$\geq 60$ & 20 & 13,3 \\
\hline \multicolumn{3}{|l|}{ Ocupação } \\
\hline Estuda & 5 & 3,4 \\
\hline Trabalha & 56 & 37,3 \\
\hline Desempregado & 66 & 44,0 \\
\hline Aposentado/pensionista & 23 & 15,3 \\
\hline \multicolumn{3}{|l|}{ Renda } \\
\hline < 1 salário mínimo & 106 & 70,7 \\
\hline 1 - 5 salários mínimos & 44 & 29,3 \\
\hline \multicolumn{3}{|l|}{ Número de moradores na residência } \\
\hline $1-3$ & 82 & 54,7 \\
\hline $4-6$ & 57 & 38,0 \\
\hline$\geq 7$ & 11 & 7,3 \\
\hline \multicolumn{3}{|l|}{ Escolaridade } \\
\hline Ensino fundamental incompleto e completo & 76 & 50,7 \\
\hline Ensino médio incompleto e completo & 52 & 34,7 \\
\hline Ensino superior incompleto e completo & 22 & 14,6 \\
\hline \multicolumn{3}{|l|}{ Cor da pele } \\
\hline Branco & 24 & 16,0 \\
\hline Pardo & 70 & 46,7 \\
\hline Negro & 56 & 37,3 \\
\hline Total & 150 & 100,0 \\
\hline
\end{tabular}

Dos indivíduos avaliados, $94 \%(n=141)$ passavam por algum grau de insegurança alimentar e nutricional. A insegurança leve foi a mais frequente, acometendo $35,3 \%(n=53)$ (Figura 1).

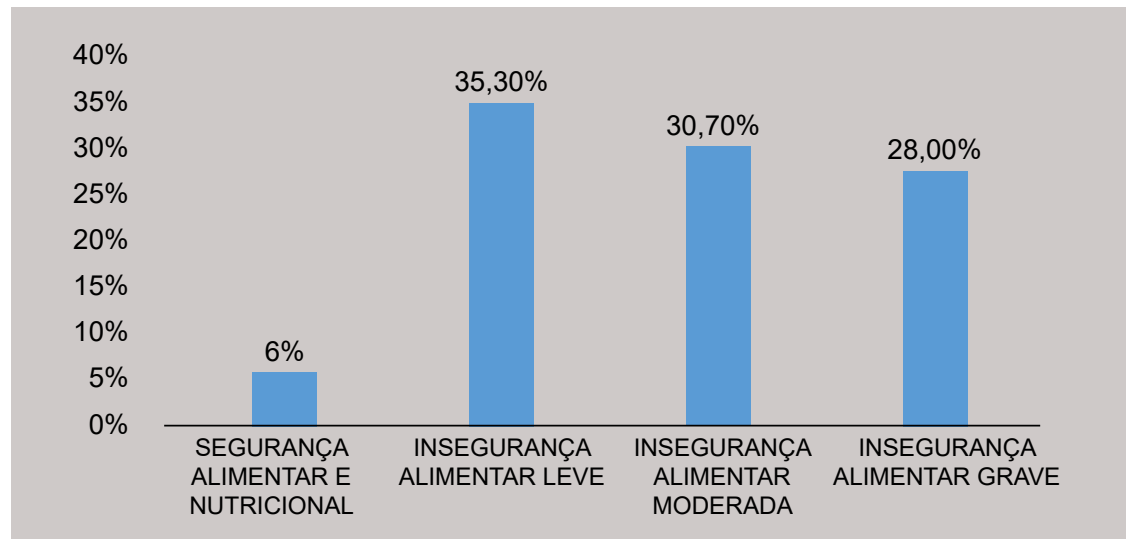

Figura 1 - Situação de (in)segurança alimentar e nutricional de moradores de uma comunidade carente. São Luís, Maranhão, 2019. 
$\mathrm{Na}$ análise não ajustada, apresentar idade de 30 a 59 anos (IRR=1,05; IC=0,98-1,12), trabalhar (IRR=0,94; $I C=0,88-1,00)$, estar desempregado (IRR=0,95; IC =0,90-1,00), ser pensionista/aposentado (IRR=0,94; IC $=0$,88$1,00)$ e ter renda familiar menor que um salário mínimo (IRR=0,86; IC=0,74-1,01) foi associado à IAN (Tabela II).

Tabela II - Análise não ajustada da associação das características sociodemográficas com a insegurança alimentar e nutricional de moradores de uma comunidade carente. São Luís, Maranhão, 2019.

\begin{tabular}{|c|c|c|c|}
\hline Variável & IRR & IC (95\%) & p-valor \\
\hline \multicolumn{4}{|l|}{ Sexo } \\
\hline Masculino & 1 & - & 1 \\
\hline Feminino & 1,03 & $(0,94-1,14)$ & 0,432 \\
\hline \multicolumn{4}{|l|}{ Estado Civil } \\
\hline Com companheiro(a) & 1 & - & 1 \\
\hline Sem companheiro (a) & 1,04 & $(0,97-1,12)$ & 0,206 \\
\hline \multicolumn{4}{|l|}{ Idade (anos) } \\
\hline $18-29$ & 1 & - & 1 \\
\hline $30-59$ & 1,05 & $(0,98-1,12)$ & 0,160 \\
\hline 60 & 0,91 & $(0,74-1,10)$ & 0,346 \\
\hline \multicolumn{4}{|l|}{ Ocupação } \\
\hline Estuda & 1 & - & 1 \\
\hline Trabalha & 0,94 & $(0,88-1,00)$ & 0,089 \\
\hline Desempregado & 0,95 & $(0,90-1,00)$ & 0,084 \\
\hline Aposentado/pensionista & 0,86 & $(0,74-1,01)$ & 0,085 \\
\hline \multicolumn{4}{|l|}{ Renda } \\
\hline 1 - 5 salários & 1 & - & 1 \\
\hline$<1$ salários & 1,07 & $(1,01-1,31)$ & 0,017 \\
\hline \multicolumn{4}{|l|}{ Número de moradores na residência } \\
\hline 1 a 3 & 1 & - & 1 \\
\hline 4 a 6 & 1,00 & $(0,92-1,09)$ & 0,834 \\
\hline 7 e mais & 0,96 & $(0,79-1,17)$ & 0,745 \\
\hline \multicolumn{4}{|l|}{ Escolaridade } \\
\hline Ensino superior incompleto e completo & 1 & - & 1 \\
\hline Ensino fundamental incompleto e completo & 1,00 & $(0,90-1,11)$ & 0,905 \\
\hline Ensino médio incompleto e completo & 0,94 & $(0,83-1,07)$ & 0,402 \\
\hline \multicolumn{4}{|l|}{ Cor da pele } \\
\hline Branco & 1 & - & 1 \\
\hline Pardo & 1,28 & $(0,89-1,17)$ & 0,681 \\
\hline Negro & 1,03 & $(0,90-1,18)$ & 0,646 \\
\hline
\end{tabular}

IRR: Risco Relativo; IC: Intervalo de Confiança 
Já na análise ajustada, observou-se que a renda menor que um salário mínimo esteve associada à situação de IAN vivida pelos indivíduos (IRR=1,07; IC=1,07-1,31), não sendo encontrada associação significativa para as demais variáveis do estudo (Tabela III).

Tabela III - Análise ajustada da associação das características sociodemográficas com a Insegurança Alimentar e Nutricional de moradores de uma comunidade carente. São Luís, Maranhão, 2019.

\begin{tabular}{lccc}
\hline Variável & IRR & IC (95\%) & p-valor \\
\hline Idade (anos) & & & \\
$18-29$ & 1 & - & 1 \\
$30-59$ & 1,05 & $(0,97-1,13)$ & 0,155 \\
$\geq 60$ & 0,95 & $(0,82-1,11)$ & 0,589 \\
Renda & & & \\
$1-5$ salários & 1 & - & 1 \\
$<1$ salário & 1,07 & $(1,01-1,31)$ & $\mathbf{0 , 0 1 7}$ \\
Ocupação & & & \\
Estuda & 1 & - & 1 \\
Trabalha & 1 & - & 1 \\
Desempregado & 0,93 & $(0,87-1,00)$ & 0,071 \\
Aposentado/Pensionista & 0,89 & $(0,78-1,00)$ & 0,057 \\
\hline
\end{tabular}

IRR: Risco Relativo; IC: Intervalo de Confiança

\section{DISCUSSÃO}

O presente estudo mostrou a elevada prevalência de IAN nas famílias investigadas, sendo mais alta que a encontrada na PNAD ${ }^{(3)}$, em que $60,9 \%$ da população maranhense estava em situação de IAN. É importante ressaltar que a comunidade pesquisada no presente estudo está localizada em uma região periférica da cidade de São Luís, Maranhão, aspecto que possivelmente influenciou no desfecho da pesquisa.

Estudos realizados na região Nordeste também destacam elevada prevalência de IAN, como a pesquisa realizada em quatro assentamentos localizados em Sergipe ${ }^{(17)}$, que revelou a existência de $88,8 \%$ das famílias, sendo $40,2 \%$ em situação de IAM e IAG. Na cidade de Teresina, Piauí, a prevalência de IAN era de $65 \%$ nas famílias assistidas pela Estratégia de Saúde da Família ${ }^{(18)}$. No município de Queimadas, Paraíba, entre as 204 famílias avaliadas, $65,4 \%$ sofriam com IAN ${ }^{(19)}$. Observou-se, em estudo realizado no Maranhão( ${ }^{(20)}$, que apenas $29,6 \%(n=283)$ das crianças apresentaram situação de segurança alimentar, e que $70,4 \%$ (673) delas se encontravam com algum tipo de insegurança alimentar.

Um estudo que avaliou a (in)segurança alimentar e nutricional, segundo indicadores socioeconômicos e nutricionais, em famílias residentes em zona rural de Minas Gerais ${ }^{(21)}$, identificou $49,4 \%$ das famílias em IAN, segundo a EBIA, concluindo que é necessário a inclusão desses indicadores em atendimentos básicos para elaborar estratégias de políticas públicas direcionadas às dificuldades encontradas.

As famílias que participaram do presente estudo possuíam algumas particularidades: metade dos indivíduos pesquisados eram adolescentes maiores de 18 anos e adultos jovens com até 29 anos que estavam desempregados, moradores vindos de outros municípios do estado do Maranhão, tinham renda familiar menor que um salário mínimo, entre outras características, que podem ter influenciado nos resultados.

O combate à IAN nos grupos mais vulneráveis faz parte dos desafios do II Plano Nacional de Segurança Alimentar e Nutricional (PLANSAN), tendo como meta constatar os grupos e territórios mais vulneráveis em SAN, realizando um mapeamento de insegurança alimentar e nutricional com o objetivo de auxiliar políticas públicas que ampliem as condições de acesso à alimentação dos que ainda se encontram vulneráveis ${ }^{(22)}$. Portanto, torna-se necessário que haja uma articulação intersetorial do PLANSAN no âmbito municipal para promoção de ações direcionadas a essa população, tendo em vista que encontramos um elevado número de famílias em insegurança alimentar na comunidade estudada.

Analisando-se os fatores associados à presença de insegurança alimentar dos indivíduos da comunidade investigada no atual estudo, a variável renda foi associada à IAN, no entanto observou-se uma associação limite 
entre a variável ocupação e a IAN. Em uma revisão sistemática com metanálise de estudos desenvolvidos no Brasil sobre SAN com a aplicação da $\mathrm{EBIA}^{(23)}$, a renda familiar foi a variável que mais se associou à IAN entre os estudos analisados.

Dos participantes investigados na presente pesquisa, a maioria recebia menos de um salário mínimo, demostrando a precária condição econômica em que vivia essa população, o que possivelmente não permitiria condições dignas de alimentação. Dados semelhantes foram encontrados em estudo realizado no município de Diamantina, Minas Gerais, o qual constatou que as famílias com renda inferior a um salário mínimo tinham 1,8 vezes mais chances de conviver com IAM e IAG ${ }^{(24)}$. Em estudo ${ }^{(25)}$ que avaliou 978 famílias de 21 municípios do estado do Maranhão e na capital São Luís, observou-se que as famílias que sobreviviam com menos de meio salário mínimo (per capita) tiveram quase duas vezes mais riscos de apresentarem insegurança alimentar. A baixa renda dificulta o acesso adequado a alimentos seguros e nutritivos ${ }^{(26)}$.

Em relação à ocupação, observou-se, na atual pesquisa, que quase metade dos entrevistados estavam desempregados, uma situação adversa para o acesso aos alimentos, ao se considerar que estar empregado é uma garantia de fonte de renda e sustento para as famílias. $\mathrm{Na}$ avaliação da associação de insegurança alimentar com condições demográficas e socioeconômicas em domicílios de Campinas, São Paulo(27), os autores identificaram que uma das condições associadas à IAL foi o chefe da família estar desempregado por mais de seis meses. Além disso, a IAM e a IAG estavam associadas à condição de emprego informal do chefe da família, diferente do resultado do presente estudo, em que não houve associação entre IAN e desemprego. A relevância da estabilidade financeira reside, entre outros aspectos, na viabilização de um acesso regular e permanente aos alimentos por todos os indivíduos ${ }^{(28)}$.

Em 2016, o Brasil assinou os Objetivos de Desenvolvimento Sustentável (ODS), que prevê, no objetivo 2, a erradicação da fome até 2030 e garantir que todas as pessoas tenham acesso a alimentos seguros, nutritivos e suficientes para todo o ano ${ }^{(29)}$. Contudo, no presente estudo, constatou-se um cenário preocupante de IAN no município de São Luís. Nesse contexto, a educação alimentar e nutricional (EAN) através do aproveitamento integral dos alimentos é uma estratégia a ser desenvolvida nessa comunidade, considerando que uma parcela importante dos entrevistados estava desempregada ou recebia menos de um salário mínimo, afetando a aquisição de alimentos em quantidade suficiente e/ou o acesso a alimentos de qualidade. Essa estratégia poderá melhorar a qualidade nutricional da alimentação dessa população ${ }^{(30)}$. Entretanto, para a correta implantação dessa ação é preciso que as famílias sejam orientadas e treinadas quanto ao aproveitamento integral dos alimentos.

Um achado que chamou atenção no presente estudo foi a associação limítrofe entre a IAN e os indivíduos aposentados/pensionistas (IRR=0,94; IC=0,88-1,00; $p<0,057$ ), decorrente, provavelmente, do fato de que a maioria das famílias depende da aposentadoria desses idosos. Para algumas famílias, a aposentadoria constitui-se em sua única fonte de renda.

Entre os entrevistados do presente estudo, foi maior o percentual de homens como chefes do domicílio, achado possivelmente associado a melhores oportunidades no mercado de trabalho para o sexo masculino, com maior renumeração e cargos de maior prestígio, ficando a cargo da mulher a função de cuidadora dos filhos e dos afazeres domésticos, acarretando em sua permanência na residência. Porém o número de famílias chefiadas por mulheres tem aumentado ${ }^{\left({ }^{11)}\right.} \mathrm{e}$, assim, na atual pesquisa, a mulher era a única responsável pela renda da família.

Em relação ao estado civil, houve maior percentual de indivíduos que viviam com companheiro na pesquisa em questão. Constatou-se que o estado civil não apresentou associação com a IAN. Esse achado vai ao encontro do trabalho realizado com famílias de pré-escolares da zona rural de um município do Vale do Paraíba paulista(32), no qual foi possível constatar que o estado civil das mães não interferiu na condição de insegurança alimentar da família. Contudo, uma pesquisa nacional desenvolvida pelo Instituto Brasileiro de Análises Sociais e Econômicas (IBASE) ${ }^{(33)}$, realizada com 5 mil titulares do programa Bolsa Família, verificou que a prevalência de IAL foi de $29,6 \%$ em famílias cujas mães possuíam cônjuge e que, entre aquelas que não tinham cônjuge, destacaram-se a IAM $(36,31 \%)$ e a IAG $(24,37 \%)$.

A idade não esteve associada à IAN na presente pesquisa, diferente do observado em pesquisa realizada no estado do Ceará(34), na qual foi encontrada associação entre insegurança alimentar e faixa etária, e IAG maior no grupo com menores de 18 anos. A presença de menores de 18 anos no domicílio influencia, estando associada à IAN, porém esta relação existe apenas quando não há um controle do efeito de outros fatores envolvidos na determinação da IAN, como o número de pessoas na residência ${ }^{(35)}$.

O presente estudo mostra que uma das características da comunidade pesquisada é possuir arranjo familiar com presença de mais de uma pessoa na residência, seguindo uma tendência das tradicionais famílias brasileiras. Nesta 
investigação, o número de moradores no domicílio não foi associado à IAN, porém, em estudo realizado para avaliar a situação de segurança alimentar e seus determinantes em municípios integrantes do Consórcio ${ }^{(9)}$, os domicílios com sete pessoas ou mais obtiveram 3,19 vezes mais chances de a família estar em situação de IAM ou IAG. Outro estudo ${ }^{(36)}$ também relacionou a insegurança alimentar ao maior número de pessoas por domicílio, resultados diferentes do encontrado no presente estudo, possivelmente por ser uma tendência atual de famílias menores. De acordo com os dados da publicação da PNAD, a família brasileira é composta, em média, por três integrantes ${ }^{(37)}$.

A escolaridade não mostrou associação com a IAN na atual pesquisa. Esse resultado se assemelha ao achado entre nutrizes atendidas em Unidades Básicas de Saúde (UBS) do município de Santa Maria, Rio Grande do Sul, no qual foi observado que a IAL e a IAM se mantiveram semelhantes, independentemente do nível de escolaridade das avaliadas ${ }^{(38)}$. Mas difere do encontrado em estudo com 243 famílias do município de Viçosa, Minas Gerais, que obteve associação entre escolaridade materna e insegurança alimentar, pois as mães que possuíam menos de 7 anos de estudo tinham um risco maior (de 1,4 vezes) de insegurança alimentar quando comparadas àquelas com mais de 7 anos de estudo ${ }^{(39)}$.

Quanto à cor da pele, a maior parte da amostra do presente estudo se autodeclarou parda ou negra. No Maranhão, segundo o IBGE, os negros são maioria, $74 \%$ da população ${ }^{(37)}$. Uma pesquisa que investigou a prevalência e fatores associados à IAN em famílias residentes em um bairro popular do município de Santo Antônio de Jesus, no Recôncavo Baiano ${ }^{(40)}$, identificou que $84,3 \%$ eram pardos e associação da IAN com cor da pele, mostrando, ainda, que a prevalência de IAN foi significativamente maior em famílias nas quais a pessoa de referência se autodeclarava preta $(p=0,037)$. Discordou-se estatisticamente deste estudo, pois não houve associação entre IAN e cor da pele no atual estudo.

É importante ressaltar algumas limitações encontradas no desenvolvimento do presente estudo. Durante a aplicação dos questionários no domicílio dos entrevistados, as respostas frequentemente eram fornecidas com alguma desconfiança e constrangimento. A fim de minimizar esse viés durante a coleta de dados, eram explicados os objetivos e a importância da pesquisa. À medida que os moradores sentiam-se mais seguros, respondiam aos questionamentos. Outra limitação a considerar refere-se ao delineamento transversal, que dificulta as análises de associação causal.

O ponto forte do estudo foi a utilização da EBIA, um instrumento adaptado para a população brasileira, de simples aplicação e análise, caracterizando as famílias em diferentes níveis de IAN. Além da fácil compreensão por parte do entrevistado e de seu baixo custo, a EBIA é utilizada frequentemente em estudos, inclusive em inquéritos nacionais, o que confere maior confiabilidade aos resultados.

Diante dos resultados encontrados, poderiam ser elaboradas estratégias, como transformar terrenos baldios em hortas comunitárias, para melhorar o cenário da IAN dessa população, haja vista as condições de vida desses indivíduos, proporcionando uma mudança do cenário de vulnerabilidade atual.

\section{CONCLUSÃO}

A prevalência de insegurança alimentar foi elevada, observando-se ser maior a prevalência de IAL, contudo a soma do percentual da insegurança alimentar moderada e grave representou metade da amostra, apontando que os participantes investigados não têm acesso aos alimentos em quantidade e qualidade adequadas às suas necessidades, demostrando a vulnerabilidade social em que vivem. A IAN mostrou-se associada à baixa renda, revelando um problema de grande magnitude que atinge os investigados.

\section{CONFLITOS DE INTERESSE}

Os autores declaram não haver conflitos de interesses.

\section{CONTRIBUIÇÕES}

Andressa Pestana Brito e Virgínia Nunes Lima participaram da elaboração e delineamento do estudo e da redação e/ou revisão do manuscrito. Eliziane Gomes da Costa Moura da Silva participou da aquisição, análise e interpretação de dados. Adriana Sousa Rêgo, Luciana Pereira Pinto Dias e Wyllyane Rayana Chaves Carvalho participaram da redação e/ou revisão do manuscrito. Josélia Dias Silva participou da elaboração e delineamento do estudo. Janaina Maiana Abreu Barbosa participou da aquisição, análise e interpretação de dados e redação e/ou revisão do manuscrito. 


\section{REFERÊNCIAS}

1. Brasil. Presidência da República. Lei $n^{\circ} 11.346$, de 15 de setembro de 2006. Cria o Sistema Nacional de Segurança Alimentar e Nutricional - SISAN com vistas em assegurar o direito humano à alimentação adequada e dá outras providências. Diário Oficial da União; 18 set. 2006.

2. Gregório MJ, Graça P, Nogueira PJ, Gomes S, Santos CA, Boavida J. Proposta Metodológica para a Avaliação da Insegurança Alimentar em Portugal. Rev Nutrícias. 2014;21:4-11.

3. Ministério do Planejamento, Orçamento e Gestão (BR); Instituto Brasileiro de Geografia e Estatística. Censo demográfico 2013. Rio de Janeiro: IBGE; 2013.

4. Ministério do Desenvolvimento Social e Combate à Fome (BR); Ministério do Planejamento, Orçamento e Gestão; Instituto Brasileiro de Geografia e Estatística. Pesquisa Nacional por Amostra de Domicílio: segurança alimentar 2013. Rio de Janeiro: IBGE; 2004.

5. Ministério do Planejamento, Orçamento e Gestão (BR); Instituto Brasileiro de Geografia e Estatística. Censo demográfico 2010. Rio de Janeiro: IBGE; 2010.

6. Pereira MHQ, Pereira MLAS, Martins BEP, Santos SMC. Segurança Alimentar e Nutricional e fatores associados em municípios baianos de diferentes portes populacionais. Rev Segur Aliment Nutr. 2019;26:1-11.

7. Morais DC, Lopes SO, Priore SE. Indicadores de avaliação da Insegurança Alimentar e Nutricional e fatores associados: revisão sistemática. Cienc Saude Colet [Internet]. 2019 [acesso em 2019 Nov 19]. Disponível em: http://www.cienciaesaudecoletiva.com.br/artigos/indicadores-de-avaliacao-da-inseguranca-alimentar-enutricional-e-fatores-associados-revisao-sistematica/17083

8. Costa NS. Prevalência e fatores associados a insegurança alimentar e nutricional [dissertação]. Maceió: Universidade Federal de Alagoas; 2016.

9. Ribeiro JCS, Santos SMC, Soares TMB, Accioly JABC. Segurança Alimentar e Nutricional: avaliação e fatores determinantes em consórcio de municípios, Bahia, Brasil. Demetra. 2018;13(1):83-100.

10. Panigassi G, Segall-Corrêa AM, Marin-León L, Pérez-Escamilla R, Sampaio MFA, Maranha LK. Insegurança alimentar como indicador de iniqüidade: análise de inquérito populacional. Cad Saúde Pública. 2008;24(10):2376-84.

11. Brasil. Lei $n^{\circ}$ 11.346, de 15 de setembro de 2006. Lei Orgânica de Segurança Alimentar e Nutricional. Institui - Sistema Nacional de Segurança Alimentar e Nutricional a fim de assegurar direito humano à alimentação adequada para toda população brasileira. Brasília, DF: Diário Oficial da União; 18 set 2006.

12. Conselho Nacional de Segurança Alimentar e Nutricional. Princípios e diretrizes para uma política nacional de segurança alimentar. Brasília: CONSEA; 2004.

13. Ministério da Saúde (BR), Secretaria de Vigilância em Saúde, Secretaria de Atenção à Saúde. Política Nacional de Promoção da Saúde: PNPS: Anexo I da Portaria de Consolidação nº 2, de 28 de setembro de 2017, que consolida as normas sobre as políticas nacionais de saúde do SUS/ Ministério da Saúde, Secretaria de Vigilância em Saúde, Secretaria de Atenção à Saúde. Brasília: Ministério da Saúde; 2018.

14. Soares MESM. Qualidade de vida e segurança alimentar: estudo de base populacional no município de João Pessoa-PB [tese]. João Pessoa: Universidade Federal da Paraíba; 2016.

15. Gubert MB, Perez-Escamilla R. Insegurança alimentar grave municipal no Brasil em 2013. Ciênc Saúde Colet. 2018;23(10):3433-44.

16. Segall-Corrêa AM, Pérez-Escamilla R, Sampaio MFA, Marín-León L, Panigassi G, Maranha LK, et al. Acompanhamento e avaliação da segurança alimentar de famílias brasileiras: validação de metodologia e de instrumento de coleta de informação Urbano/Rural. Campinas: UNICAMP; 2004.

17. Almeida JA, Santos AS, Nascimento MAO, Oliveira JVC, Silva DG, Mendes-Netto RS. Fatores associados ao risco de insegurança alimentar e nutricional em famílias de assentamentos rurais. Ciênc Saúde Colet. 2017;22(2):479-88.

18. Sabóia RCB, Santos MM. Prevalência de insegurança alimentar e fatores associados em domicílios cobertos pela Estratégia Saúde da Família em Teresina, Piauí, 2012-2013. Epidemiol Serv Saúde. 2015;24(4):749-58. 
19. Pedraza DF, Bezerra TA, Ana C, Cerqueira DR, Fonsêca JS. (In)-Segurança alimentar de famílias residentes em um município do interior da Paraíba, Brasil. Rev Saude Publica. 2017;19(5):649-56.

20. Lopes AF, Frota MTBA, Leone C, Szarfarc SC. Perfil nutricional de crianças no estado do maranhão. Rev Bras Epidemiol. 2019(22):e190008.

21. Morais DC, Sperandio N, Dutra LV, Franceschini SCC, Santos RHS, Priore SE. Indicadores socioeconômicos, nutricionais e de percepção de insegurança alimentar e nutricional em famílias rurais. Segur Aliment Nutr. 2018;25(2):1-11.

22. Câmara Interministerial de Segurança Alimentar e Nutricional (BR). II Plano Nacional de Segurança Alimentar e Nutricional - PLANSAN 2016-2019. Brasília: CAISAN; 2018.

23. Bezerra TA, Olinda, RA, Pedraza, DF. Insegurança alimentar no Brasil segundo diferentes cenários sociodemográficos. Ciênc Saúde Colet. 2017;22(2):637-51.

24. Santos KKD, Mandacaru SMP, Matta RA, Murta NMG, Freitas RF, Lessa AC. Fatores associados à insegurança alimentar em populações pobres do município de Diamantina-MG. Revista Espacios. 2017;38(12)19.

25. Rocha NP, Szarfarc SC, Lira PIC, Sequeira LAS, Silveira VNC, Frota MTBA. Condição de (In)Segurança Alimentar e fatores associados de famílias com crianças menores de cinco anos de idade do Estado do Maranhão. Segur Aliment Nutr. 2018;25(3):71-80.

26. Amaral VR, Basso D. Segurança Alimentar e Nutricional no Brasil: uma análise em perspectiva histórica. Rev Desenvolv Reg. 2016;13(1):181-200.

27. Souza BFNJ, Marin-Leon L, Camargo DFM, Segall-Corrêa AM. Demographic and socioeconomic conditions associated with food insecurity in households in Campinas, SP, Brazil. Rev Nutr. 2016;29(6):845-57.

28. Omuemu VO, Otasowie EM, Onyiriuka U. Prevalence of food insecurity in Egor local government area of Edo State, Nigeria. Ann Afri Med. 2012;11(3):139-45.

29. Organização das Nações Unidas. Transformando nosso mundo: Agenda 2030 para o desenvolvimento Sustentável. 2015 [acesso em 2016 Mar 29]. Disponível em: https://nacoesunidas.org/pos2015/agenda2030/

30. Cardoso F, Avelar KES. Aproveitamento integral dos alimentos e o seu impacto na saúde. Sustentabilidade Destaque. 2015;6(3):131-43.

31. Cavenhagi S, Alves JED. Mulheres chefes de família no Brasil: avanços e desafios. Rio de Janeiro: ENSCPES; 2018.

32. Rodrigues AM, Santos EC, Faria TP, Faria AL, Chamon EMQO. Segurança alimentar de famílias com préescolares da zona rural de um município do Vale do Paraíba paulista. DEMETRA. 2020;15:1-12.

33. Instituto Brasileiro de Análises Sociais e Econômicas. Repercussões do Programa Bolsa Família na segurança alimentar e nutricional: relatório síntese. Rio de Janeiro: IBASE; 2008.

34. Costa LNF, Braga MM, Rocha M, Lima MS, Campêlo WF, Vasconcelos CMCS. Fatores associados à insegurança alimentar em pessoas que vivem com HIVIAIDS. Rev Bras Promoç Saúde. 2018;31(1):1-8.

35. Hoffmann R. Determinantes da Insegurança Alimentar no Brasil: Análise dos Dados da PNAD de 2004. Segur Aliment Nutr. 2008;15(1):49-61.

36. Maas NM, Sassi M, Andres R, Meucci, Rodrigo Dalke, Cesar J. Insegurança Alimentar em famílias de área rural do extremo sul do Brasil. Cienc Saude Colet [Internet]. 2018 [acesso em 2019 Nov 19]. Disponível em: http://www.cienciaesaudecoletiva.com.br/artigos/inseguranca-alimentar-em-familias-de-area-rural-doextremo-sul-do-brasil/17030?id=17030

37. Ministério do Planejamento, Orçamento e Gestão (BR); Instituto Brasileiro de Geografia e Estatística. Censo demográfico 2012. Rio de Janeiro: IBGE; 2012.

38. Silva KSM, Fontoura ES, Blümke AC, Margutti KMM. Insegurança alimentar e sua relação com fatores socioeconômicos de nutrizes atendidas na atenção primária. Rev Eletrônica Disciplinarum Scientia. 2015;16(2):221-9. 
39. Sperandio N, Priore SE. Prevalência de insegurança alimentar domiciliar e fatores associados em famílias com pré-escolares, beneficiárias do Programa Bolsa Família de Viçosa, Minas Gerais, Brasil. Epidemiol Serv Saúde. 2015;24(4):739-48.

40. Oliveira DS, Demétrio F, Soares MD, Henrique FCS, Santos LA. Prevalência e fatores associados à insegurança alimentar. Rev Baiana Saúde Pública. 2017;41(3):561-79.

\section{Endereço para correspondência:}

Andressa Pestana Brito

Universidade Ceuma - Campus Renascença

Rua Josué Montello, 1

Bairro: Renascença II

CEP: 65075-120 - São Luís - MA - Brasil

E-mail: andressa.britto.ab@gmail.com

Como citar: Brito AP, Lima VN, Silva EGCM, Rêgo AS, Dias LPP, Silva JD, et al. Fatores associados à insegurança alimentar e nutricional em comunidade carente. Rev Bras Promoç Saúde. 2020;33:10415. 